\title{
Body mass index influences the plasma glucose concentration during iatrogenic hypoglycemia in people with type 2 diabetes mellitus: A cross-sectional study
}

\author{
Po Chung Cheng ${ }^{1}$, Shang Ren Hsu ${ }^{1}$, Shih Te Tu ${ }^{\text {Corresp.. }}{ }^{1}$, Yun Chung Cheng ${ }^{2}$, Yu Hsiu Liu ${ }^{3}$ \\ 1 Division of Endocrinology and Metabolism, Department of Internal Medicine, Changhua Christian Hospital, Changhua City, Taiwan \\ 2 Department of Radiology, Taichung Veterans General Hospital, Taichung, Taiwan \\ 3 Department of Accounting and Information Systems, National Taichung University of Science and Technology, Taichung, Taiwan \\ Corresponding Author: Shih Te Tu \\ Email address: 10836@cch.org.tw
}

Background. Hypoglycemia occurs in an appreciable number of individuals with type 2 diabetes mellitus (T2DM) who are receiving glycemic therapy. latrogenic hypoglycemia induces not only complications but also substantial medical expense. Intervention for relevant risk factors may help avert severe hypoglycemia and enhance quality of life in atrisk individuals. This study investigates the relationship between body mass index (BMI) and plasma glucose concentration during iatrogenic hypoglycemia in people with T2DM. Methods. Enrollment criteria were people above 20 years of age, with existing diagnosis of T2DM, a documented plasma glucose level $\leq 70 \mathrm{mg} / \mathrm{dL}$, and acute cognitive impairment requiring hospitalization. Participants were classified into two groups according to their BMI. Specifically, lower BMI subgroup denotes individuals whose BMI fall within lower half of the study population, and vice versa. Plasma glucose concentration, length of hospital stay, and serum electrolyte level at hospitalization were compared between these BMI subgroups. Moreover, multivariate regression analysis was performed to identify covariates associated with plasma glucose level during iatrogenic hypoglycemia. Results. This study enrolled 107 participants for whom 54 were assigned to a higher BMI subgroup and the remainder to a lower BMI subgroup. People with lower BMI harbored substantially reduced plasma glucose concentration during iatrogenic hypoglycemia compared to those with higher BMI $(30.1 \pm 9.6 \mathrm{mg} / \mathrm{dL}$ vs. $38.4 \pm 12.3 \mathrm{mg} / \mathrm{dL}, P<0.001)$. Nonetheless, the length of stay ( $6.2 \pm 4.6$ days vs. $5.7 \pm 4.0$ days, $P=0.77$ ) and serum potassium level (3.7 $\pm 0.9 \mathrm{meq} / \mathrm{L}$ vs. $3.9 \pm 0.8 \mathrm{meq} / \mathrm{L}, P=0.14$ ) were comparable between subgroups. Multivariate regression analysis identified $\mathrm{BMI}$ as a determinant of plasma glucose concentration in diabetic individuals with iatrogenic hypoglycemia ( $\beta$ coefficient: $0.72, P=$ 0.008). Discussion. In individuals with T2DM who experience severe iatrogenic hypoglycemia, BMI influences the plasma glucose level at hospitalization. People with 
lower BMI harbored appreciably reduced plasma glucose concentration relative to their higher BMI counterparts. In lower weight people, therefore, appropriate dosing of antidiabetic medications, frequent self-monitoring of blood glucose level and adequate nutritional support may help avert more severe hypoglycemia. Overall, BMI potentially influences the severity of iatrogenic hypoglycemia in people with T2DM. 
1

2

3 Body mass index influences the plasma glucose concentration during iatrogenic hypoglycemia in

4

5

6

7

$8{ }^{1}$ Division of Endocrinology and Metabolism, Department of Internal Medicine, Changhua

9 Christian Hospital, 135 Nanxiao St., Changhua City, Changhua County, Taiwan

10

11 Section 4, Taichung, Taiwan

12 and Technology, 129 San Min Rd., Taichung, Taiwan

14

15

16

17 Email address: 10836@cch.org.tw

18

19

20

21

22

23

24

Author cover page people with type 2 diabetes mellitus: A cross-sectional study Po Chung Cheng ${ }^{1}$, Shang Ren Hsu ${ }^{1}$, Shih Te Tu${ }^{1}$, Yun Chung Cheng ${ }^{2}$, Yu Hsiu Liu ${ }^{3}$ 2 Department of Radiology, Taichung Veterans General Hospital, 1650 Taiwan Boulevard

${ }^{3}$ Department of Accounting and Information Systems, National Taichung University of Science

Corresponding author: Shih Te Tu

Email address. 10836@cch.org.tw

18


26

27

28

29

30

31

32

33

34

Abstract

Background. Hypoglycemia occurs in an appreciable number of individuals with type 2 diabetes mellitus (T2DM) who are receiving glycemic therapy. Iatrogenic hypoglycemia induces not only complications but also substantial medical expense. Intervention for relevant risk factors may help avert severe hypoglycemia and enhance quality of life in at-risk individuals. This study investigates the relationship between body mass index (BMI) and plasma glucose concentration during iatrogenic hypoglycemia in people with T2DM.

Methods. Enrollment criteria were people above 20 years of age, with existing diagnosis of T2DM, a documented plasma glucose level $\leq 70 \mathrm{mg} / \mathrm{dL}$, and acute cognitive impairment requiring hospitalization. Participants were classified into two groups according to their BMI. Specifically, lower BMI subgroup denotes individuals whose BMI fall within lower half of the study population, and vice versa. Plasma glucose concentration, length of hospital stay, and serum electrolyte level at hospitalization were compared between these BMI subgroups. Moreover, multivariate regression analysis was performed to identify covariates associated with plasma glucose level during iatrogenic hypoglycemia.

Results. This study enrolled 107 participants for whom 54 were assigned to a higher BMI subgroup and the remainder to a lower BMI subgroup. People with lower BMI harbored substantially reduced plasma glucose concentration during iatrogenic hypoglycemia compared to those with higher BMI $(30.1 \pm 9.6 \mathrm{mg} / \mathrm{dL}$ vs. $38.4 \pm 12.3 \mathrm{mg} / \mathrm{dL}, P<0.001)$. Nonetheless, the length of stay $(6.2 \pm 4.6$ days vs. $5.7 \pm 4.0$ days, $P=0.77)$ and serum potassium level $(3.7 \pm 0.9$ meq/L vs. $3.9 \pm 0.8$ meq $/ \mathrm{L}, P=0.14)$ were comparable between subgroups. Multivariate regression analysis identified BMI as a determinant of plasma glucose concentration in diabetic 
49 individuals with iatrogenic hypoglycemia ( $\beta$ coefficient: $0.72, P=0.008$ ).

50 Discussion. In individuals with T2DM who experience severe iatrogenic hypoglycemia, BMI

51 influences the plasma glucose level at hospitalization. People with lower BMI harbored

52 appreciably reduced plasma glucose concentration relative to their higher BMI counterparts. In

53 lower weight people, therefore, appropriate dosing of antidiabetic medications, frequent self-

54 monitoring of blood glucose level and adequate nutritional support may help avert more severe

55 hypoglycemia. Overall, BMI potentially influences the severity of iatrogenic hypoglycemia in 56 people with T2DM.

57

58

59

60

61

62

63

64

65

66

67

68

69

70

71

72 
73 Body mass index influences the plasma glucose concentration during iatrogenic hypoglycemia in

74

75

76

77

78

79

80

81

82

83

84

85

86

87

88

89

90

91

92

93

94

95

96

people with type 2 diabetes mellitus: A cross-sectional study

Introduction

Hypoglycemia occurs in an appreciable number of individuals with type 2 diabetes mellitus (T2DM) who are receiving antidiabetic therapy (Shafiee et al., 2012). Iatrogenic hypoglycemia induces not only complications but also substantial medical expense related to hospitalization (Rhee et al., 2016). Prevention of hypoglycemia in diabetes is therefore an integral component of treatment (Clayton, Woo \& Yale, 2013). Importantly, intervention for relevant risk factors may reduce severe hypoglycemia and enhance quality of life in people with T2DM.

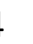

Iatrogenic hypoglycemia is associated with clinical complications. Elderly people with recurrent hypoglycemia are at risk of cognitive impairment (McNay \& Cotero, 2010). Severe hypoglycemia also contributes to a higher incidence of cardiovascular event and mortality (Hanefeld, Frier \& Pistrosch, 2016). Moreover, risk of traumatic injury is notably increased in patients who experience severe hypoglycemia (Kachroo et al., 2015).

1 Epidemiologic studies have identified several risk factors for hypoglycemia in people with 2 T2DM. Stringent glycemic targets are closely linked to the incidence of hypoglycemia (Lipska et 3 al., 2013). In addition, severe hypoglycemia occurs more frequently in the elderly, presumably

94 due to lack of symptom recognition (Abdelhafiz et al., 2015). Studies have also implicated 5 diabetes duration as a risk factor, as demonstrated by progressive deterioration of hypoglycemic 96 counterregulatory mechanism in people with longstanding disease (Dailey et al., 2013; Amiel et 
97 al., 2008).

98

99 The body mass index (BMI), as defined by dividing the weight in kilograms by the square of the

100 height in meters, may be an important but overlooked risk factor for severe hypoglycemia.

101 People with T2DM who have a lower BMI are also likely to have lower hepatic glycogen stores

102 and this can diminish the secretion of glucose counterregulatory hormones during hypoglycemia

103 (Winnick et al., 2016). Reduced counterregulatory hormones may compromise an individual's

104 capacity to stabilize blood glucose level during fasting (Izumida et al., 2013). Furthermore, lower

105 BMI in elderly individuals may reflect underlying frailty (Lee et al., 2014), which impairs both

106 the recognition of and behavioral defense against hypoglycemia.

107

108 This study investigates the relationship between BMI and plasma glucose concentration during 109 iatrogenic hypoglycemia in people with T2DM.

110

111 Materials and Methods

112

113 This is a cross-sectional study conducted at Changhua Christian Hospital in central Taiwan.

114 Individuals hospitalized at the Endocrinology ward from September 2011 to August 2017 were

115 assessed for eligibility. Enrollment criteria were people above 20 years of age, with existing

116 diagnosis of T2DM, a documented plasma glucose level $\leq 70 \mathrm{mg} / \mathrm{dL}$ (Cryer, 2015), and acute

117 cognitive impairment that required hospitalization.

118

119 Candidates were excluded if they had chronic infection, traumatic injury, acute cerebro- or 120 cardiovascular event, organ failure, or whose hypoglycemia was considered independent of 
121 antidiabetic medications. Moreover, people with malignancy, eating disorders, and previous

122 gastrointestinal surgery were ineligible. The study was approved by the Institutional Review

123 Board of Changhua Christian Hospital (CCH IRB number: 171105). Written consent to

124 participate in the study was provided by the patients' next of kin.

125

126 Participants were assigned to two equally sized subgroups according to their BMI. Specifically,

127 lower BMI subgroup denotes individuals whose BMI fall within lower half of the study

128 population, and vice versa. Blood tests were performed at hospitalization except for glycosylated

129 hemoglobin $\mathrm{A}_{1 \mathrm{c}}\left(\mathrm{HbA}_{1 \mathrm{c}}\right)$, which was extracted from existing laboratory data prior to the

130 hypoglycemic event. Serum HbA1c was measured by ion-exchange high-performance liquid

131 chromatography using BioRad VARIANT ${ }^{\mathrm{TM}}$ II Turbo system. Serum biochemistry including

132 glucose, creatinine, and potassium were measured by Beckman Coulter UniCel DxC 800

133 Synchron ${ }^{\mathrm{TM}}$ Clinical Systems. The analytical precision for serum glucose is within $2 \mathrm{mg} / \mathrm{dL}$.

134 Prescription details were collected from electronic medical records. The enrollment process is

135 illustrated in Figure 1.

136

137 Demographic data between BMI subgroups were compared using Mann-Whitney U test for

138 continuous variables and Pearson's $\chi^{2}$ test for categorical variables. The plasma glucose

139 concentration, serum potassium, and length of hospital stay were compared using Mann-

140 Whitney U test. Multivariate regression analysis was performed to identify predictors of plasma

141 glucose level at hospitalization. A two-tailed $P$ value of less than 0.05 indicated statistical

142 significance. Analysis was performed using IBM SPSS version 22.0 (IBM SPSS Statistics for

143 Windows, Armonk, NY). 
145 Results

146

147 The study enrolled 107 participants with T2DM who were hospitalized due to severe iatrogenic

148 hypoglycemia. As shown in Table 1, demographic characteristics including age (76.5 \pm 13.8

149 years vs. $73.8 \pm 11.7$ years, $P=0.172)$, preceding $\mathrm{HbA}_{1 \mathrm{c}}(6.7 \pm 1.1 \%$ vs. $6.8 \pm 1.3 \%, P=0.52)$,

150 duration of diabetes $(7.6 \pm 4.5$ years vs. $9.3 \pm 4.9$ years, $P=0.051)$, and serum creatinine $(1.6 \pm$

$1511.0 \mathrm{mg} / \mathrm{dL}$ vs. $1.9 \pm 1.6 \mathrm{mg} / \mathrm{dL}, P=0.336$ ) were comparable between BMI subgroups. Moreover,

152 the proportion of participants using antidiabetic medication commonly implicated in iatrogenic

153 hypoglycemia, such as insulin $(26 \%$ vs. $32 \%, P=0.31)$ or sulfonylurea $(65 \%$ vs. $64 \%, P=0.55)$,

154 were also similar.

155

156 As demonstrated in Table 2, participants with lower BMI harbored substantially reduced plasma

157 glucose concentration compared to those with higher BMI during iatrogenic hypoglycemia (30.1

$158 \pm 9.6 \mathrm{mg} / \mathrm{dL}$ vs. $38.4 \pm 12.3 \mathrm{mg} / \mathrm{dL}, P<0.001)$. Nonetheless, individuals with lower BMI did not

159 have an appreciably longer length of stay compared to their higher weight counterparts $(6.2 \pm 4.6$

160 days vs. $5.7 \pm 4.0$ days, $P=0.77)$. Furthermore, mean serum potassium levels were similar

161 between subgroups $(3.7 \pm 0.9 \mathrm{meq} / \mathrm{L}$ vs. $3.9 \pm 0.8 \mathrm{meq} / \mathrm{L}, P=0.14)$.

162

163 Multivariate regression analysis identified covariates that potentially influence the plasma

164 glucose concentration at hospitalization. The standardized coefficient of each independent

165 variable is listed in Table 3. As can be seen, BMI was significantly related to plasma glucose

166 concentration during iatrogenic hypoglycemia in people with T2DM ( $\beta$ coefficient: $0.72, P=$

167 0.008) after adjusting for confounding variables. 
Discussion

170

171 People with T2DM are vulnerable to the detrimental effect of hypoglycemia, which may become

172 a limiting factor in antidiabetic therapy (Seaquist et al., 2013). Apart from requiring the

173 assistance of caregivers, severe hypoglycemia also induces harmful cardiac arrhythmia and

174 functional brain failure (Chow et al., 2014). Moreover, hypoglycemia-associated autonomic

175 failure can impair the physiologic and behavioral defense against a subsequent hypoglycemic

176 event (Cryer, 2013).

177

178 The observation in this study that lower weight people with T2DM had reduced plasma glucose

179 concentration during iatrogenic hypoglycemia may be attributable to attenuated glucose

180 counterregulatory mechanisms. People with lower BMI, perhaps reflecting less availability of

181 hepatic glycogen, may have diminished secretion of glucagon and epinephrine (Winnick et al.,

182 2016), resulting in inadequate hepatic glucose production during iatrogenic hypoglycemia.

183 Furthermore, effect of glucagon may be compromised in people with inadequate glycogen since

184 this hormone raises blood glucose level through hepatic glycogenolysis (Melmed et al., 2016).

185

186 Moreover, unintentional weight loss may reflect frailty and functional disability (Xue, 2011). In

187 lower weight people, delayed recognition of hypoglycemia may partly explain their appreciably

188 lower plasma glucose level at hospitalization. People with lower BMI may therefore have limited

189 ability to counteract hypoglycemia due to reduced secretion of glucose counterregulatory

190 hormones, as discussed previously, and functional disability that leads to hypoglycemic

191 unawareness.

192 
193 Adipose tissue modifies insulin sensitivity through the production of adipokines (Waki \&

194 Tontonoz, 2007; Fasshauer \& Bluher, 2015). Weight loss improves insulin sensitivity by

195 decreasing free fatty acid mobilization and by changing adipokine profile in obesity (Schenk et

196 al., 2009; Greco et al., 2014). Moreover, intentional weight loss in T2DM correlates with lower

197 fasting plasma glucose concentration (Wing et al., 2011). Weight loss is therefore an established

198 risk factor for iatrogenic hypoglycemia in diabetes (Melmed et al., 2016). In clinical practice,

199 BMI may indirectly mirror an individual's insulin sensitivity and subsequent risk of

200 hypoglycemia. Therefore, dynamic change in body weight during glycemic treatment may

201 require a corresponding adjustment in therapeutic regimen.

202

203 Intriguingly, although participants with lower BMI harbored reduced plasma glucose level at

204 hospitalization, length of stay and serum potassium level were similar to their higher BMI

205 counterparts. In other words, hypoglycemia may not cause immediately perceivable

206 complications. Nonetheless, plasma glucose level below $30 \mathrm{mg} / \mathrm{dL}$ has been linked to permanent

207 brain injury in an animal model (Oyer, 2013), and lower weight people in this study with a mean

208 blood glucose close to this level were at risk of long-term neurologic damage.

209

210 Several implications arise from the study's finding that lower weight participants had reduced

211 plasma glucose level during iatrogenic hypoglycemia. Hypoglycemia in diabetes involves a

212 combination of therapeutic insulin excess and compromised physiologic defense (Cryer, 2013).

213 Appropriate dosing of antidiabetic medications, especially sulfonylurea and insulin (Heller et al.,

214 2007), is prudent for people with lower BMI. Lower weight people may also benefit from less

215 stringent treatment target, frequent self-monitoring of blood glucose (SMBG), and continuous

216 glucose monitoring (CGM) to detect asymptomatic hypoglycemia (Cryer, 2014). Nutritional 
217 support to increase hepatic glycogen in at risk individuals may enhance physiologic defense

218 against more severe hypoglycemia (Décombaz et al., 2011). Moreover, since the glucose-raising

219 efficacy of glucagon is unreliable in people with inadequate glycogen, an alternative method may

220 be necessary to restore normoglycemia in underweight patients.

221

222 The design of this study has limitations. To be hospitalized for treatment, participants obviously

223 circumvented lethal complications such as cardiac arrhythmia, which may lead to selection bias.

224 Furthermore, participants may have initially received management for hypoglycemia at home,

225 and blood tests at hospitalization may not reveal the lowest plasma glucose concentration. A

226 longer observation time may be necessary to identify potential complications associated with

227 severe hypoglycemia. Hypoglycemic risk may also relate to the dose of antidiabetic medications,

228 which was not addressed by the study. Moreover, neither hepatic glycogen quantity nor

229 counterregulatory hormone level was measured, both of which may influence the severity of

230 iatrogenic hypoglycemia.

231

232 Conclusions

233

234 Lower weight individuals with T2DM harbored reduced plasma glucose concentration during 235 iatrogenic hypoglycemia. People with lower BMI may have compromised defense against 236 iatrogenic hypoglycemia due to reduced secretion of counterregulatory hormones and functional 237 disability. Appropriate dosing of antidiabetic medications, individualized treatment target, 238 frequent SMBG and CGM technology may help avert more severe hypoglycemia in people with

239 lower BMI. Ultimately, nutritional support to increase hepatic glycogen may defend lower 240 weight patients against severe hypoglycemia. 
242 Acknowledgement

243

244 None.

245

246 References

247

248

Abdelhafiz AH, Rodríguez-Mañas L, Morley JE, Sinclair AJ. 2015. Hypoglycemia in older 249

people - a less well recognized risk factor for frailty. Aging Dis 6:156-167. DOI:

250

10.14336/AD.2014.0330.

251

Amiel SA, Dixon T, Mann R, Jameson K. 2008. Hypoglycaemia in type 2 diabetes. Diabet

252

Med 25: 245-254. DOI: 10.1111/j.1464-5491.2007.02341.x.

253

Clayton D, Woo V, Yale JF. 2013. Hypoglycemia. Can J Diabetes 37 Suppl 1: S69-71. DOI:

254

10.1016/j.jcjd.2013.01.022.

255

Cryer PE. 2013. Hypoglycemia in diabetes: Pathophysiology, prevalence and prevention, 2nd Edition. American Diabetes Association: Alexandria, VA.

257 Cryer PE. 2013. Mechanisms of hypoglycemia-associated autonomic failure in diabetes. $N$ Engl $258 J$ Med 369: 362-372. DOI: 10.1056/NEJMra1215228.

259 Cryer PE. 2014. Glycemic goals in diabetes: trade-off between glycemic control and iatrogenic 260 hypoglycemia. Diabetes 63: 2188-2195. DOI: 10.2337/db14-0059.

261 Cryer PE. 2015. Minimizing hypoglycemia in diabetes. Diabetes Care 38: 1583-1591. DOI: $26210.2337 / \mathrm{dc} 15-0279$.

263 Dailey GE, Gao L, Aurand L, Garg SK. 2013. Impact of diabetes duration on hypoglycaemia 264 in patients with type 2 diabetes treated with insulin glargine or NPH insulin. Diabetes Obes 
265

266

267 Fructose and galactose enhance postexercise human liver glycogen synthesis. Med Sci Sports 268 Exerc 43:1964-1971. DOI: 10.1249/MSS.0b013e318218ca5a.

269 Fasshauer M, Blüher M. 2015. Adipokines in health and disease. Trends Pharmacol Sci 270 36:461-470. DOI: 10.1016/j.tips.2015.04.014.

271 Greco M, Chiefari E, Montalcini T, Accattato F, Costanzo FS, Pujia A, Foti D, Brunetti A, 272 Gulletta E. 2014. Early effects of a hypocaloric, Mediterranean diet on laboratory parameters in 273 obese individuals. Mediators Inflamm. 2014:750860. DOI: 10.1155/2014/750860.

274 Hanefeld M, Frier BM, Pistrosch F. 2016. Hypoglycemia and cardiovascular risk: Is there a 275 major link? Diabetes Care 39 Suppl 2: S205-209. DOI: 10.2337/dcS15-3014.

276

277

278

279

280

281

282

283

284

285

286

287

288

Metab 15:1085-1092. DOI: 10.1111/dom.12131.

Décombaz J, Jentjens R, Ith M, Scheurer E, Buehler T, Jeukendrup A, Boesch C. 2011.

Heller SR, Choudhary P, Davies C, Emery C, Campbell MJ, Freeman J, Amiel SA, Malik

R, Frier BM, Allen KV, Zammitt NN, Macleod K, Lonnen KF, Kerr D, Richardson T,

Hunter S, Mclaughlin D. 2007. Risk of hypoglycaemia in types 1 and 2 diabetes: effects of treatment modalities and their duration. Diabetologia 50: 1140-1147.

Izumida Y, Yahagi N, Takeuchi Y, Nishi M, Shikama A, Takarada A, Masuda Y, Kubota

\section{M, Matsuzaka T, Nakagawa Y, Iizuka Y, Itaka K, Kataoka K, Shioda S, Niijima A,} Yamada T, Katagiri H, Nagai R, Yamada N, Kadowaki T, Shimano H. 2013. Glycogen

shortage during fasting triggers liver-brain-adipose neurocircuitry to facilitate fat utilization. Nat Commun 4: 2316. DOI: 10.1038/ncomms3316.

Kachroo S, Kawabata H, Colilla S, Shi L, Zhao Y, Mukherjee J, Iloeje U, Fonseca V. 2015. Association between hypoglycemia and fall-related events in type 2 diabetes mellitus: Analysis of a U.S. commercial database. J Manag Care Spec Pharm 21: 243-253.

Lee Y, Kim J, Han ES, Ryu M, Cho Y, Chae S. 2014. Frailty and body mass index as 
289 predictors of 3-year mortality in older adults living in the community. Gerontology 60:475-482.

290 DOI: 10.1159/000362330.

291 Lipska KJ, Warton EM, Huang ES, Moffet HH, Inzucchi SE, Krumholz HM, Karter AJ.

292 2013. $\mathrm{HbA}_{1 \mathrm{c}}$ and risk of severe hypoglycemia in type 2 diabetes: the Diabetes and aging study.

293 Diabetes Care 36(11): 3535-3542. DOI: 10.2337/dc13-0610.

294 McNay EC, Cotero VE. 2010. Mini-review: Impact of recurrent hypoglycemia on cognitive and 295 brain function. Physiol Behav 100: 234-238. DOI: 10.1016/j.physbeh.2010.01.004.

296 Melmed S, Polonsky KS, Larsen PR, Kronenberg HM. 2016. Williams Textbook of

297 Endocrinology, 13th Edition. Elsevier.

298 Oyer DS. 2013. The science of hypoglycemia in patients with diabetes. Current Diabetes

299 Reviews 9: 195-208.

300

Rhee SY, Hong SM, Chon S, Ahn KJ, Kim SH, Baik SH, Park YS, Nam MS, Lee KW, Woo

301

JT, Kim YS. 2016. Hypoglycemia and medical expenses in patients with type 2 diabetes

302

mellitus: An analysis based on the Korea National Diabetes Program Cohort. PLoS One 11(2):

303

e0148630. DOI: 10.1371/journal.pone.0148630.

304

305

306

307

308

309

310

311

312

Rodriguez H, Rosenzweig J, Vigersky R. 2013. Hypoglycemia and diabetes: A report of a workgroup of the American Diabetes Association and the Endocrine Society. Diabetes Care 36: 1384-1395. DOI: $10.2337 / \mathrm{dc} 12-2480$.

Schenk S, Harber MP, Shrivastava CR, Burant CF, Horowitz JF. 2009. Improved insulin sensitivity after weight loss and exercise training is mediated by a reduction in plasma fatty acid mobilization, not enhanced oxidative capacity. J Physiol 587: 4949-4961. DOI:

10.1113/jphysiol.2009.175489

Seaquist ER, Anderson J, Childs B, Cryer P, Dagogo-Jack S, Fish L, Heller SR, Chow E, Bernjak A, Williams S, Fawdry RA, Hibbert S, Freeman J, Sheridan PJ, Heller SR. 2014. 
313 Risk of cardiac arrhythmias during hypoglycemia in patients with type 2 diabetes and

314 cardiovascular risk. Diabetes 63: 1738-1747. DOI: 10.2337/db13-0468.

315 Shafiee G, Mohajeri-Tehrani M, Pajouhi M, Larijan B. 2012. The importance of

316 hypoglycemia in diabetic patients. J Diabetes Metab Disord 11: 17. DOI: 10.1186/2251-6581-

$317 \quad 11-17$.

318 Waki H, Tontonoz P. 2007. Endocrine functions of adipose tissue. Annu Rev Pathol 2:31-56.

319 Wing RR, Lang W, Wadden TA, Safford M, Knowler WC, Bertoni AG, Hill JO, Brancati

320 FL, Peters A, Wagenknecht L. 2011. Benefits of modest weight loss in improving

321 cardiovascular risk factors in overweight and obese individuals with type 2 diabetes. Diabetes

322 Care. 34:1481-1486. DOI: 10.2337/dc10-2415.

323 Winnick JJ, Kraft G, Gregory JM, Edgerton DS, Williams P, Hajizadeh IA, Kamal MZ,

324 Smith M, Farmer B, Scott M, Neal D, Donahue EP, Allen E, Cherrington AD. 2016. Hepatic

325 glycogen can regulate hypoglycemic counterregulation via a liver-brain axis. J Clin Invest 126:

326 2236-2248. DOI: 10.1172/JCI79895.

327 Xue QL. 2011. The frailty syndrome: Definition and natural history. Clin Geriatr Med 27: 1-15.

328 DOI: 10.1016/j.cger.2010.08.009. 
Figure 1

Enrollment process of the study

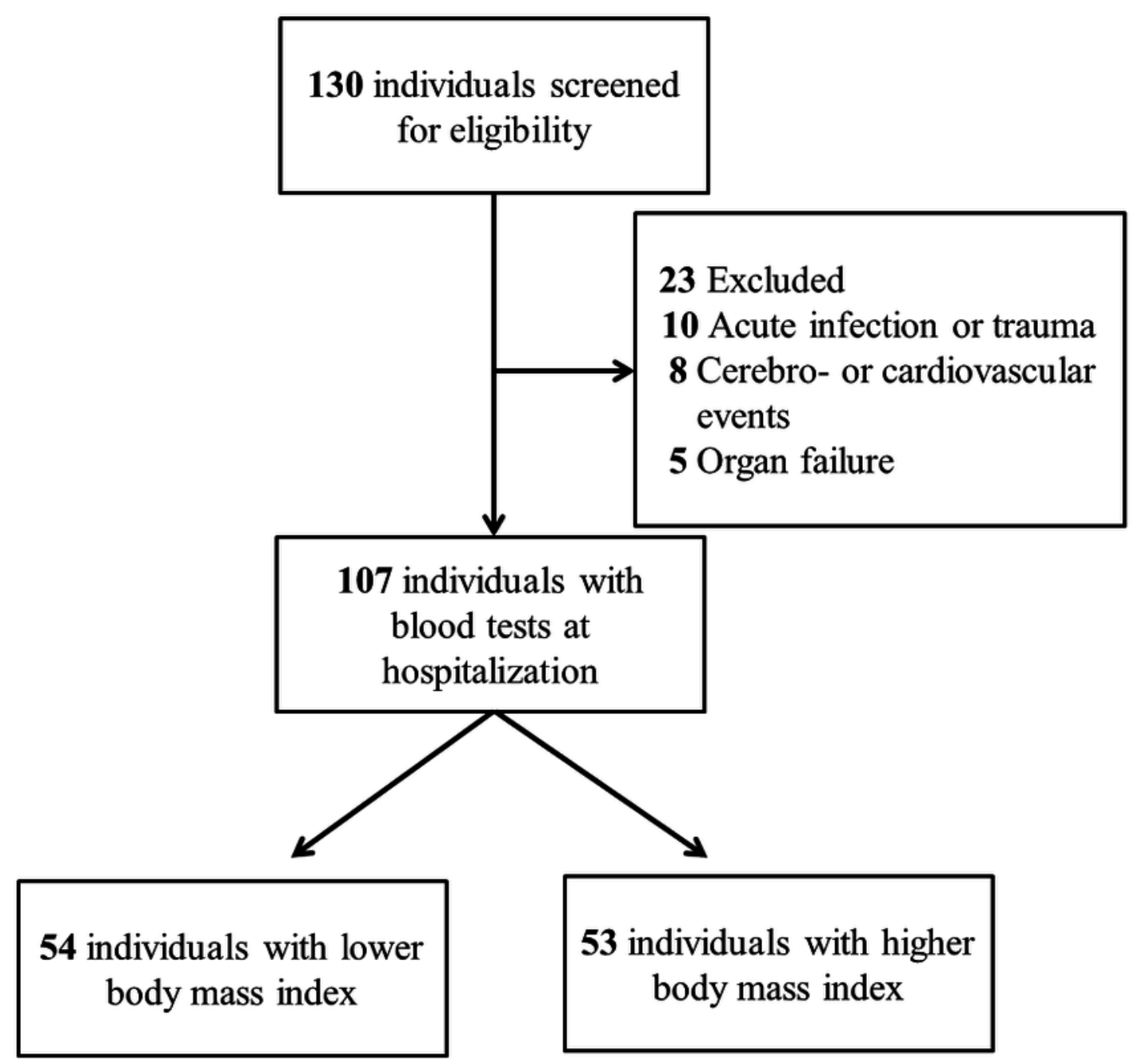




\section{Table $\mathbf{1}$ (on next page)}

Demographic characteristics of the body mass index subgroups

Data are expressed as mean with standard deviation for continuous variables and number (\%) for categorical variables. $\mathrm{BMI}$ : body mass index, $\mathrm{HbA}_{1 \mathrm{c}}$ : glycated hemoglobin $\mathrm{A}_{1 \mathrm{c}}$ 
Table 1. Demographic characteristics of the body mass index subgroups

\begin{tabular}{|c|c|c|c|}
\hline Variables & $\begin{array}{c}\text { Lower BMI } \\
(n=54)\end{array}$ & $\begin{array}{c}\text { Higher BMI } \\
(n=53)\end{array}$ & $P$ value \\
\hline Age (years) & $76.5 \pm 13.8$ & $73.8 \pm 11.7$ & 0.172 \\
\hline Sex (Female) & $29(54 \%)$ & $29(55 \%)$ & 0.53 \\
\hline BMI $\left(\mathrm{kg} / \mathrm{m}^{2}\right)$ & $20.1 \pm 1.78$ & $27.0 \pm 3.90$ & $<0.001$ \\
\hline $\mathrm{HbA}_{1 \mathrm{c}}(\%)$ & $6.7 \pm 1.1$ & $6.8 \pm 1.3$ & 0.52 \\
\hline Creatinine (mg/dL) & $1.60 \pm 1.00$ & $1.91 \pm 1.63$ & 0.336 \\
\hline $\begin{array}{l}\text { Duration of } \\
\text { diabetes } \\
\text { (years) }\end{array}$ & $7.6 \pm 4.5$ & $9.3 \pm 4.9$ & 0.051 \\
\hline Use of insulin & $14(26 \%)$ & $17(32 \%)$ & 0.31 \\
\hline Use of sulfonylurea & $35(65 \%)$ & $34(64 \%)$ & 0.55 \\
\hline \multicolumn{4}{|c|}{$\begin{array}{l}\text { Data are expressed as mean with standard deviation for continuous variables and } \\
\text { number (\%) for categorical variables. Continuous variables were compared using the } \\
\text { Mann-Whitney U test for independent samples. }\end{array}$} \\
\hline
\end{tabular}




\section{Table 2 (on next page)}

Clinical features of the body mass index subgroups

Data are expressed as mean with standard deviation for continuous variables. BMI: body mass index 
Table 2. Clinical features of the body mass index subgroups

\begin{tabular}{lccc}
\hline Variables & $\begin{array}{c}\text { Lower BMI } \\
(\boldsymbol{n}=\mathbf{5 4})\end{array}$ & $\begin{array}{c}\text { Higher BMI } \\
(\boldsymbol{n}=\mathbf{5 3})\end{array}$ & P value \\
\hline $\begin{array}{l}\text { Plasma glucose } \\
\text { concentration } \\
(\mathrm{mg} / \mathrm{dL})\end{array}$ & $30.1 \pm 9.61$ & $38.4 \pm 12.3$ & $<0.001$ \\
& & & \\
Length of stay (days) & $6.2 \pm 4.6$ & $5.7 \pm 4.0$ & 0.77 \\
Potassium (mEq/L) & $\mathbf{3 . 7} \pm \mathbf{0 . 8 6}$ & $\mathbf{3 . 9} \pm \mathbf{0 . 8 0}$ & 0.14 \\
\hline
\end{tabular}

Data are expressed as mean with standard deviation for continuous variables.

Continuous variables were compared using the Mann-Whitney U test for independent samples.

BMI: body mass index 


\section{Table 3(on next page)}

Multivariate regression analysis of covariates associated with plasma glucose concentration during iatrogenic hypoglycemia

BMI: body mass index, $\mathrm{HbA}_{1 c}$ : glycated hemoglobin $\mathrm{A}_{1 c}$ 
Table 3. Multivariate regression analysis of covariates associated with plasma glucose concentration during iatrogenic hypoglycemia

\begin{tabular}{lcc}
\hline Covariates & $\boldsymbol{\beta}$ coefficient & $\boldsymbol{P}$ value \\
\hline Age (years) & 0.014 & 0.88 \\
$\mathrm{BMI}\left(\mathrm{kg} / \mathrm{m}^{2}\right)$ & 0.72 & 0.008 \\
$\mathrm{HbA}_{\mathrm{lc}}(\%)$ & -1.18 & 0.26 \\
$\mathrm{Creatinine}(\mathrm{mg} / \mathrm{dL})$ & -1.1 & 0.21 \\
$\begin{array}{l}\text { Duration of diabetes } \\
\text { (years) }\end{array}$ & -0.037 & 0.88 \\
\hline
\end{tabular}

BMI: body mass index, $\mathrm{HbA}_{1 \mathrm{c}}$ : glycated hemoglobin $\mathrm{A}_{\mathrm{lc}}$ 